\title{
Lymphatic targeting drug delivery system and tumor treatment
}

\author{
Yu Xiao* \\ Department of Pharmacy , Sichuan University, Sichuan, 610041, China \\ *Corresponding Author email: 1075996759@qq.com
}

\begin{abstract}
Keywords: Lymphatic, Drug delivery system, Tumor therapy
\end{abstract}
Abstract. Research Objective: This study aims at observing the clinical effect of the application of the lymphatic targeting drug delivery system in the treatment of malignant tumors.

Research Methods: The patients with advanced gastric cancer in our hospital are taken as the samples. According to different treatment methods, they were randomly divided into two groups, namely the observation group and the control group. The patients in the control group were treated with fluorouracil, while the patients in the observation group were treated with compound fluorouracil liposome through the application of the lymphatic targeting drug delivery system in the treatment process.

Research Results: In the observation group, a total of six patients experienced adverse reactions, including five patients with nausea and vomiting and one patient with leukopenia, and the incidence rate of adverse reactions was $18.75 \%$. The patients' five-year survival rate was $75 \%$, and the quality of life was $86.32 \pm 2.09$ points. Compared with the control group, the data differences were statistically significant $(\mathrm{p}<0.05)$.

Research Conclusions: The application of the lymphatic targeting drug compound fluorouracil liposome in the treatment of gastric cancer is of great clinical value for increasing the patients' disease control rate, improving the patients’ living quality and prolonging the patients' life.

\section{Introduction}

Malignant tumors are a major disease that endangers human health. The conventional treatment methods are based on radical surgery combined with radiotherapy and chemotherapy [1-5], which can prolong the patients' life to a certain extent but can lead to patients' more adverse reactions and lower living quality [6]. Some studies have pointed out that the application of the lymphatic targeting drug delivery system in the treatment of malignant tumors has achieved favorable results [7-8]. This paper randomly selects 64 patients from the patients with gastric cancer treated in our hospital from August 2015 to August 2016 as the samples, observing the treatment effect of the lymphatic targeting drug delivery system.

\section{Data and methods}

\subsection{General data}

The patients with advanced gastric cancer in our hospital are taken as the samples. According to different treatment methods, they were randomly divided into two groups, namely the observation group and the control group. There were 32 patients aged between 34 and 85 in the observation group, including 16 male patients and 16 female patients, with the average age of $51.20 \pm 0.87$, whereas there were 32 patients aged between 40 and 88 in the control group, including 17 male patients and 15 female patients, with the average age of $51.21 \pm 0.92$. The comparisons of the patients in the two groups can be made ( $\mathrm{p}>0.05)$.

\subsection{Methods}

The patients in the control group were treated with fluorouracil. The method was as follows: the patients were treated with fluorouracil $750 \mathrm{mg} / \mathrm{d} 1--5+$ cisplatin $20 \mathrm{mg} / \mathrm{d} 1--5+$ mitomycin $10 \mathrm{mg} / \mathrm{d} 1$.

The patients in the observation group were treated with compound fluorouracil liposome through the application of the lymphatic targeting drug delivery system in the treatment process. The usage 
and dosage was as follows: (1) the patients were continuously treated with 5--7d-compound fluorouracil liposome $80--120 \mathrm{mg} / \mathrm{d}$; (2) the patients were treated with the intravenous injection of cisplatin 20mg/d1--5+mitomycin 10mg/d1.

1.3 Observing indicators

The disease control rate, the incidence rate of adverse reactions, the one-year survival rate and the living quality of the patients in the two groups were respectively observed. The living quality is scored by the SF-36 scale. The higher score indicates the higher living quality.

1.4 Determination standards for the disease control rate

CR: Target lesions have disappeared, or there have been no new lesions for at least 4 weeks. PR: Target lesions have decreased by 30\% or more than 30\% for at least 4 weeks. SD: Target lesions have not decreased or increased. PD: Target lesions have increased by $20 \%$ or over $20 \%$, or there have been new lesions. The disease control rate $=(C R+P R+S D) / 32 \times 100 \%$.

1.5 Statistical method

SPSS 23.0 software is adopted to process the data. $x 2$ test is used for counting, which is represented by (\%), while t test is used for measurement, which is denoted by (mean \pm standard deviation). $\mathrm{P}<0.05$ means that the data differences are statistically significant.

\section{Result}

2.1 Disease control rate of two groups of patients

2.1.1 Disease control rate of observation group

There are 32 patients in observation group, and 28 patients are good controller of disease, which accounts for $\mathbf{8 7 . 5 \%}$ in disease control rate, details are listed in table1:

Table1 Disease control rate of patients in obsevation group

\begin{tabular}{cccccc}
\hline Item & CR (n/\%) & PR (n/\%) & SD (n/\%) & PD (n/\%) & $\begin{array}{c}\text { control rate } \\
(\mathrm{n} / \%)\end{array}$ \\
\hline Data(n=32) & $10(31.25)$ & $15(46.88)$ & $3(9.38)$ & $4(12.50)$ & $28(87.50)$ \\
\hline
\end{tabular}

2.1.2 Disease control rate of patients in control group

There are 32 patients in control group, and 29 patients are good controller of disease ,which accounts for $90.63 \%$ in disease control rate. In contrast to ovation group,no statistically significant differences are detected.

Table2 Disease control rate of patients in control group

\begin{tabular}{cccccc}
\hline Item & CR (n/\%) & PR (n/\%) & SD (n/\%) & PD (n/\%) & $\begin{array}{c}\text { Control rate } \\
(\mathrm{n} / \%)\end{array}$ \\
\hline Date(n=32) & $10(31.25)$ & $18(56.25)$ & $1(3.13)$ & $3(9.38)$ & $29(90.63)$ \\
\hline
\end{tabular}

2.2 Adverse effects rate of two groups of patients

2.2.1 Adverse effects rate of patients in observation group

There are 6 patients that appear adverse effects in observation group,in which 5 patients are nausea and vomiting and 1 is leukopenia, and the adverse effects rate is $18.75 \%$,details are listed in table3:

Table 3 Adverse effects rate of patients in observation group

\begin{tabular}{ccccc}
\hline Item & $\begin{array}{c}\text { Nausea and } \\
\text { vomiting } \\
(\mathrm{n} / \%)\end{array}$ & $\begin{array}{c}\text { Leukopenia } \\
(\mathrm{n} / \%)\end{array}$ & $\begin{array}{c}\text { Myocardial } \\
\text { ischemia } \\
(\mathrm{n} / \%)\end{array}$ & $\begin{array}{c}\text { Total } \\
(\mathrm{n} / \%)\end{array}$ \\
\hline Date $(\mathrm{n}=32)$ & $5(15.63)$ & $1(3.13)$ & $0(0)$ & $6(18.75)$ \\
\hline
\end{tabular}




\subsubsection{Adverse effects rate of patients in control group}

There are 15 patients that appear adverse effects in control group, in which 12 patients are Nausea and vomiting and 1 is Leukopenia,and the adverse effects rate is $46.88 \%$,details are listed in table 4 :

Table 4 Adverse effects rate of patients in control group

\begin{tabular}{ccccc}
\hline Item & $\begin{array}{c}\text { Nausea and } \\
\text { vomiting } \\
(\mathrm{n} / \%)\end{array}$ & $\begin{array}{c}\text { Leukopenia } \\
(\mathrm{n} / \%)\end{array}$ & $\begin{array}{c}\text { Myocardial } \\
\text { ischemia } \\
(\mathrm{n} / \%)\end{array}$ & $\begin{array}{c}\text { Total } \\
(\mathrm{n} / \%)\end{array}$ \\
\hline Data $(\mathrm{n}=32)$ & $12(37.50)$ & $2(6.25)$ & $1(3.13)$ & $15(46.88)$ \\
\hline
\end{tabular}

2.3 One-year survival rate and the living quality of the patients in the two groups

One-year survival rate of observation group is $75 \%$, and living quality scores ( $86.32 \pm 2.09$. In contrast to the control group,the data differences are statistically significant. Details are listed in table 5:

Table5 One-year survival rate and the living quality of the patients in the two groups

Group Number(n) 5-year survival rate Living quality

(scores)

\begin{tabular}{cccc}
\hline observation group & 32 & $24(75.00)$ & $86.32 \pm 2.09$ \\
Control group & 32 & $13(40.63)$ & $65.73 \pm 1.84$ \\
$\mathrm{p}$ & - & $<0.05$ & $<0.05$ \\
\hline
\end{tabular}

\section{Discussion}

The lymphatic targeting drug delivery system is one of the treatment methods for malignant tumors ${ }^{[9-10]}$. In the current drug delivery method for malignant tumors, drugs are often absorbed orally and enter the cardiovascular system ${ }^{[11]}$. When the above drug delivery method is adopted, drugs mostly need to be metabolized firstly through the livers and intestines, resulting in the low drug utilization rate and numerous adverse reactions. As indicated from this study, when the patients with gastric cancer were treated with fluorouracil, a total of 15 patients experienced adverse reactions, including 12 patients with nausea and vomiting, 2 patients with leucopenia and 1 patient with myocardial ischemia, and the incidence rate of adverse reactions was $46.88 \%$. Through the observation of the prognosis of the patients, it is seen that in this group, the patients' five-year survival rate was $40.63 \%$, and the living quality was $65.73 \pm 1.84$ points. The process of metastasis and proliferation of malignant cells is the process of transferring cells to lymph nodes. Therefore, if the lymphatic targeting drug delivery can be conducted, the drugs will directly act on the metastasis, so that the control rate of malignant tumors can be increased ${ }^{[12-15]}$. Lymph is lipophilic, and treating the patients with malignant tumors with liposome or niosome drugs is the main idea of the current lymphatic targeting drug delivery. Compound fluorouracil liposome is a kind of lymphatic targeting drugs. After entering the human body orally, the drugs can enter the assembly process of intestinal lipoproteins and be preferentially transported to the intestinal lymph. Compared with treating the patients only with fluorouracil, treating the patients with compound fluorouracil liposome can make the drugs pertinently act on the lymphatic metastasis, so as to achieve the purpose of killing the tumor cells and inhibiting the progress of the disease. As indicated from this study, after the patients with gastric cancer were treated with the lymphatic targeting drug compound fluorouracil liposome, a total of six patients experienced adverse reactions, including five patients with nausea and vomiting and one patient with leukopenia, and the incidence rate of adverse reactions was $18.75 \%$. Compared with the treatment with fluorouracil, the incidence rate of the patients' adverse reactions was lower, and the safety of medication was higher. In this group, the patients' five-year survival rate was $75 \%$, and 
the living quality was $86.32 \pm 2.09$ points. Compared with the treatment with fluorouracil, the prognosis of the patients was better $(\mathrm{p}<0.05)$. Through observing the disease control rate of the patients in the two groups, it is seen that the data differences in the disease control rate between the two drug delivery methods were not statistically significant $(P>0.05)$. It is suggested that the use of the lymphatic targeting drug compound fluorouracil liposome in the treatment of gastric cancer has achieved remarkable treatment effects. Specifically, it can effectively reduce the incidence rate of various adverse reactions, improve the prognosis of the patients and prolong the life of the patients.

In summary, the application of the lymphatic targeting drug compound fluorouracil liposome in the treatment of gastric cancer is of great clinical value for increasing the patients' disease control rate, improving the patients' living quality and prolonging the patients' life.

\section{References}

[1] Jain V, Jain S, Mahajan S C. Nanomedicines Based Drug Delivery Systems For Anti-Cancer Targeting And Treatment.[J]. Current Drug Delivery, 2015, 12(2):177-191.

[2] Zhu D, Tao W, Zhang H, et al. Docetaxel (DTX)-loaded polydopamine-modified TPGS-PLA nanoparticles as a targeted drug delivery system for the treatment of liver cancer.[J]. Acta Biomaterialia, 2016, 30:144-154.

[3] Wei Y, Zhou F, Zhang D, et al. A graphene oxide based smart drug delivery system for tumor mitochondria-targeting photodynamic therapy[J]. Nanoscale, 2016, 8(6):3530-3538.

[4] Borrajo E, Abellan-Pose R, Soto A, et al. Docetaxel-loaded polyglutamic acid-PEG nanocapsules for the treatment of metastatic cancer[J]. Journal of Controlled Release, 2016, 238:263-271.

[5] Si J, Shao S, Shen Y, et al. Macrophages as Active Nanocarriers for Targeted Early and Adjuvant Cancer Chemotherapy[J]. Small, 2016, 12(37):5108-5119.

[6] Mao Y, Li X, Chen G, et al. Thermosensitive Hydrogel System with Paclitaxel Liposomes Used in Localized Drug Delivery System for In Situ Treatment of Tumor: Better Antitumor Efficacy and Lower Toxicity[J]. Journal of Pharmaceutical Sciences, 2015, 105(1):194-204.

[7] Merlot A M, Sahni S, Lane D J, et al. Potentiating the cellular targeting and anti-tumor activity of Dp44mT via binding to human serum albumin: two saturable mechanisms of Dp44mT uptake by cells.[J]. Oncotarget, 2015, 6(12):10374-10398.

[8] Dong X, Wang W, Qu H, et al. Targeted delivery of doxorubicin and vincristine to lymph cancer: evaluation of novel nanostructured lipid carriers in vitro and in vivo.[J]. Drug Delivery, 2015, 23(4):1374-1378.

[9] Long T N, Yang X Z, Du X, et al. Enhancing tumor-specific intracellular delivering efficiency of cell-penetrating peptide by fusion with a peptide targeting to EGFR[J]. Amino Acids, 2015, 47(5):997-1006.

[10] Pang M F, Georgoudaki A M, Lambut L, et al. TGF-|[beta]|1-induced EMT promotes targeted migration of breast cancer cells through the lymphatic system by the activation of CCR7|[sol]|CCL21-mediated chemotaxis[J]. Oncogene, 2016, 35(6):748-760.

[11] Mammadovabach E, Mangin P, Lanza F, et al. Platelets in cancer. From basic research to therapeutic implications.[J]. Hamostaseologie, 2015, 35(4):325-336.

[12] Shamsi M, Sedaghatkish A, Dejam M, et al. Magnetically assisted intraperitoneal drug delivery for cancer chemotherapy.[J]. Drug Delivery, 2018, 25(1):846-861. 
[13] Hruby S, Englberger C, Lusuardi L, et al. Fluorescence - guided targeted pelvic Lymph node dissection in intermediate and high risk prostate cancer[J]. Journal of Urology, 2015, 194(2):357-363.

[14] Tardi P, Wan C P, Mayer L. Passive and Semi-Active Targeting of Bone Marrow and Leukemia Cells Using Anionic Low Cholesterol Liposomes[J]. Journal of Drug Targeting, 2016, 24(9):1-26.

[15] Wang X G, Dong Z Y, Cheng H, et al. A multifunctional metal-organic framework based tumor targeting drug delivery system for cancer therapy[J]. Nanoscale, 2015, 7(38):16061-16070. 